\title{
The convergence condition of a series appearing in connection problems and the determination of Stokes' multipliers
}

\author{
By \\ Mitsuhiko КонNo
}

In order to seek 'the solutions in the large' of ordinary differential equations with irregular singular point of rank 1 , which have the following reduced forms, i.e.,

$$
t \frac{d X}{d t}=(A+t B) X,
$$

we had to solve a two point connection problem between one set of fundamental solutions in the neighbourhood of regular singular point $t=0$, expressed by the convergent series as follows,

$$
X_{j}(t)=t^{\rho_{j}} \sum_{m=0}^{\infty} G_{j}(m) t^{m}
$$

and another set of fundamental solutions in the sectorial neighbourhood of irregular singular point $t=\infty$, expressed by the following asymptotic forms, i.e.,

$$
X^{k}(t)=e^{\lambda_{k} t} t^{a_{k k}} \sum_{s=0}^{\infty} H^{k}(s) t^{-s}
$$

where the series in the right hand side are the formal solutions.

In the analysis of solving the connection problem, it is important to investigate the structure of the coefficients $G_{j}(m)$, which satisfy the following linear difference equations

$$
\left\{\begin{array}{l}
\left(\rho_{j}+m-A\right) G_{j}(m)=B G_{j}(m-1), \\
\left(\rho_{j}-A\right) G_{j}(0)=0 .
\end{array}\right.
$$

Then, in order to solve the above difference equations, we reduced 
the difference equations to the differential equations

$$
\left\{\varepsilon-\frac{1}{\lambda_{k}}\left(\lambda_{k}-B\right)\right\} \frac{d F^{k}(\varepsilon, m)}{d \varepsilon}=\left(\rho_{j}+m-A\right) F_{j}^{k}(\varepsilon, m)
$$

and obtained, using the theory of the differential equations, the general solutions of the difference equations as follows,

$$
F_{j}^{k}(1, m)=\sum_{s=0}^{\infty} H^{k}(s) g_{j}^{k}(m+s) .
$$

What concerns this method is described in detail in the papers $[1],[2],[3]$.

In this paper, we will obtain by the very simple method, which is based on the estimation of the coefficients $H^{k}(s)$ of the formal solutions, the sufficient condition for the convergence of the series $\sum_{s=0}^{\infty} H^{k}(s) g_{j}^{k}(m+s)$. We will describe it in section 1 and show, using the result of section 1 , how to determine Stokes' multipliers of the two point connection problem for a system of ordinary differential equations in section 2 .

The aim of this paper is to show the possibility of weakening the pentagonal condition.

1. Now we investigate the convergence of the series $\sum_{s=0}^{\infty} H^{k}(s) g_{j}^{k}(m+s)$. Here, the column vectors $H^{k}(s)(k=1,2, \cdots, n)$ satisfy the following difference equations

$$
\left\{\begin{array}{l}
\left(a_{k^{k}}-s-A\right) H^{k}(s)=\left(B-\lambda_{k}\right) H^{k}(s+1) \\
\left(B-\lambda_{k}\right) H^{k}(0)=0,
\end{array}\right.
$$

where $a_{k k}$ is the $k$-th diagonal element of the matrix $A$, and $\lambda_{k}$ is the $k$-th eigenvalue of the diagonal matrix $B$ such that $\lambda_{j} \neq \lambda_{k}(j \neq k)$.

The scalar functions $g_{j}^{k}(m+s)(k=1,2, \cdots, n ; j=1,2, \cdots, n)$ are defined by

$$
g_{j}^{k}(m+s)=\frac{\lambda_{k}^{m+s} \Gamma\left(\rho_{j}-a_{k k}\right)}{\Gamma\left(m+s+\rho_{j}-a_{k k}+1\right)}=\frac{\lambda_{k}^{m+s} \Gamma(\beta-1)}{\Gamma(m+s+\beta)},
$$

where $\rho_{j}$ is the $j$-th eigenvalue of the matrix $A$ and $\beta=\rho_{j}-a_{k k}+1$. It is assumed that $\beta-1=\rho_{j}-a_{k k} \neq$ non-positive integers $(j=1,2, \cdots, n$; 
$k=1,2, \cdots, n)$. We define the matrix norm and the vector norm as follows.

$$
\begin{aligned}
& \|A\|=\max _{j} \sum_{k=1}^{n}\left|a_{j k}\right|, \\
& \|v\|=\max _{j}\left|v_{j}\right| .
\end{aligned}
$$

Then we have the following properties of the norms. Let $v, w$ be the column vectors and $A$ be the matrix.

$$
v=0 \text { if and only if }\|v\|=0 .
$$

(ii) Let $c$ be a complex scalar. $\|c v\|=|c|\|v\|$.

(iii) $\quad\|v+w\| \leqq\|v\|+\|w\|$

(iv) $\quad \lim _{n \rightarrow \infty} v_{n}=0$ if and only if $\lim _{n \rightarrow \infty}\left\|v_{n}\right\|=0$

( v ) $\quad\|A v\| \leqq\|A\|\|v\|$

Now we assume that $\|A\|>0$ and take an integer $N$ which is larger than $\|A\|$.

We divide the series into two parts.

$$
\sum_{s=0}^{\infty} H^{k}(s) g_{j}^{k}(m+s)=\sum_{s=0}^{N-1} H^{k}(s) g_{j}^{k}(m+s)+\sum_{s=N}^{\infty} H^{k}(s) g_{j}^{k}(m+s) .
$$

In order to obtain the convergence of the series, we only may prove the convergence of the second part of the series. We set

$$
H^{k}(s)=\left(\begin{array}{c}
h_{1}^{k}(s) \\
h_{2}^{k}(s) \\
\vdots \\
h_{n}^{k}(s)
\end{array}\right) .
$$

Then we can rewrite the difference equations (1) in componentwise as follows.

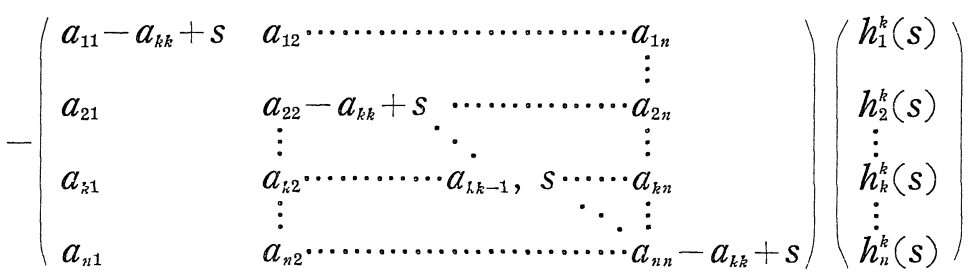




$$
=\left(\begin{array}{ccc}
\lambda_{1}-\lambda_{k} & & \\
\lambda_{2}-\lambda_{k} & & \\
\ddots & & \\
0 & & \ddots \\
& & \lambda_{n}-\lambda_{k}
\end{array}\right)\left(\begin{array}{c}
h_{1}^{k}(s+1) \\
h_{2}^{k}(s+1) \\
\vdots \\
h_{k}^{k}(s+1) \\
\vdots \\
\dot{h}_{n}^{k}(s+1)
\end{array}\right)=\left(\begin{array}{c}
\left(\lambda_{1}-\lambda_{k}\right) h_{1}^{k}(s+1) \\
\left(\lambda_{2}-\lambda_{k}\right) h_{2}^{k}(s+1) \\
\vdots \\
0 \\
\vdots \\
\left(\lambda_{n}-\lambda_{k}\right) h_{n}^{k}(s+1)
\end{array}\right) .
$$

The $k$-th component of the vector $H^{k}(s)$, i.e., $h_{k}^{k}(s)$ is determined by

$$
\begin{aligned}
-s h_{k}^{k}(s)= & a_{k 1} h_{1}^{k}(s)+a_{k 2} h_{2}^{k}(s)+\cdots+a_{k k-1} h_{k-1}^{k}(s) \\
& +a_{k k+1} h_{k+1}^{k}(s)+\cdots+a_{k n} h_{n}^{k}(s),
\end{aligned}
$$

and if, $s \geqq N>\|A\|$, we will show that the absolute value of the $k$-th component $h_{k}^{k}(s)$ is less than the norm $\left\|H^{k}(s)\right\|$. For if $\left|h_{k}^{k}(s)\right|$ is equal to the norm $\left\|H^{k}(s)\right\|$, we obtain

$$
\begin{aligned}
s\left|h_{k}^{k}(s)\right| & \leqq\left|a_{k 1}\right|\left|h_{1}^{k}(s)\right|+\cdots+\left|a_{k k-1}\right|\left|h_{k-1}^{k}(s)\right| \\
& +\left|a_{k k+1}\right|\left|h_{k+1}^{k}(s)\right|+\cdots+\left|a_{k n}\right|\left|h_{n}^{k}(s)\right| \\
& \leqq\left(\left|a_{k 1}\right|+\cdots+\left|a_{k k-1}\right|+\left|a_{k k+1}\right|+\cdots+\left|a_{k n}\right|\right)\left\|H^{k}(s)\right\| \\
& \leqq\|A\|\left\|H^{k}(s)\right\|=\|A\|\left|h_{k}^{k}(s)\right|,
\end{aligned}
$$

but this inequality contradicts the assumption $s \geqq N>\|A\|$. Next we estimate the $j$-th component of the column vector $H^{k}(s+1)$.

$$
\begin{aligned}
& -\left(\lambda_{j}-\lambda_{k}\right) h_{j}^{k}(s+1)=a_{j 1} h_{1}^{k}(s)+a_{j 2} h_{2}^{k}(s)+\cdots \\
& +\left(a_{j j}-a_{k k}+s\right) h_{j}^{k}(s)+\cdots+a_{j n} h_{n}^{k}(s) \\
& \left|\lambda_{j}-\lambda_{k}\right|\left|h_{j}^{k}(s+1)\right| \leqq\left|a_{j 1}\right|\left|h_{1}^{k}(s)\right|+\cdots \\
& +\left\{\left|a_{j j}\right|+\left|a_{k k}\right|+s\right\}\left|h_{j}^{k}(s)\right|+\cdots+\left|a_{j_{n}}\right|\left|h_{n}^{k}(s)\right| \\
& \leqq \max _{j}\left|h_{j}^{k}(s)\right|\left\{\left|a_{j 1}\right|+\cdots+\left|a_{j_{n}}\right|+\left|a_{k k}\right|+s\right\} \\
& \leqq\left\|H^{k}(s)\right\|\left\{\|A\|+\left|a_{k k}\right|+s\right\} \text {. }
\end{aligned}
$$

Here we set

$$
\begin{aligned}
& \left|\lambda-\lambda_{k}\right|=\min _{j \neq k}\left|\lambda_{j}-\lambda_{k}\right|>0 \quad(j=1,2, \cdots, n), \\
& \alpha=\|A\|+\left|a_{k k}\right| .
\end{aligned}
$$

Then we obtain

$$
\left|h_{j}^{k}(s+1)\right| \leqq \frac{1}{\left|\lambda-\lambda_{k}\right|}\left\|H^{k}(s)\right\|(s+\alpha), \quad(j \neq k) .
$$


Whence, if $s \geqq N$, we can estimate the norm $\left\|H^{k}(s+1)\right\|$ by the norm $\left\|H^{k}(s)\right\|$ as follows.

$$
\left\|H^{k}(s+1)\right\| \leqq \frac{1}{\left|\lambda-\lambda_{k}\right|}\left\|H^{k}(s)\right\|(s+\alpha), \quad s \geqq N .
$$

So, by using the formula (4), we obtain

$$
\begin{aligned}
\left\|H^{k}(s+N)\right\| & \leqq \frac{\left\|H^{k}(N)\right\|}{\left|\lambda-\lambda_{k}\right|^{s}}(\alpha+N)(\alpha+N+1) \cdots(\alpha+N+s-1) \\
& =\frac{\left\|H^{k}(N)\right\|}{\left|\lambda-\lambda_{k}\right|^{s}} \frac{\Gamma(s+\alpha+N)}{\Gamma(\alpha+N)} .
\end{aligned}
$$

Now we estimate the second part of the series (3), using the above estimation (5).

$$
\begin{aligned}
& \left\|\sum_{s=N}^{\infty} H^{k}(s) g_{j}^{k}(m+s)\right\| \leqq \sum_{s=N}^{\infty}\left\|H^{k}(s)\right\|\left|g_{j}^{k}(m+s)\right| \\
& =\sum_{s=0}^{\infty}\left\|H^{k}(s+N)\right\|\left|g_{j}^{k}(m+N+s)\right| \\
& \leqq \sum_{s=0}^{\infty} \frac{\left\|H^{k}(N)\right\|}{\left|\lambda-\lambda_{k}\right|^{s}} \frac{\Gamma(s+N+\alpha)}{\Gamma(\alpha+N)} \frac{\left|\lambda_{k}\right|^{s+m+N}|\Gamma(\beta-1)|}{|\Gamma(s+m+N+\beta)|} \\
& =\frac{\left\|H^{k}(N)\right\||\Gamma(\beta-1)|}{\Gamma(\alpha+N)}\left|\lambda_{k}\right|^{m+N} \sum_{s=0}^{\infty}\left(\frac{\left|\lambda_{k}\right|}{\left|\lambda-\lambda_{k}\right|}\right)^{s} \frac{\Gamma(s+N+\alpha)}{|\Gamma(s+m+N+\beta)|} .
\end{aligned}
$$

The radius of convergence of the last series is equal to one, considering that the series is the power series of $\left|\frac{\lambda_{k}}{\lambda-\lambda_{k}}\right|$.

So, in order to obtain the convergent series $\sum_{s=0}^{\infty} H^{k}(s) g_{j}^{k}(m+s)$, it is sufficient that $\left|\frac{\lambda_{k}}{\lambda-\lambda_{k}}\right|<1$, and this condition is again the pentagonal condition, i.e.,

$$
\left|\lambda_{k}\right|<\left|\lambda_{j}-\lambda_{k}\right| \quad(j \neq k) .
$$

The above proof is a different and simple proof of the proposition 3.3 in the paper [1], and the lemma 4.4 in the paper [2].

Now we investigate the last series of (6) in detail and can expect the convergence of the series on the circle of convergence $\left|\frac{\lambda_{k}}{\lambda-\lambda_{k}}\right|=1$.

We set $u_{s}=\left|\frac{\lambda_{k}}{\lambda-\lambda_{k}}\right|^{s} \frac{\Gamma(s+\alpha+N)}{|\Gamma(s+m+N+\beta)|}$. Then when $\left|\frac{\lambda_{k}}{\lambda-\lambda_{k}}\right|=1$, 


$$
\begin{aligned}
\frac{u_{s+1}}{u_{s}} & =\left|\frac{\lambda_{k}}{\lambda-\lambda_{k}}\right|\left|\frac{s+\alpha+N}{s+m+N+\beta}\right| \\
& =\left|1+\frac{\alpha-\beta-m}{s+m+N+\beta}\right|=1+\frac{\operatorname{Re}(\alpha-\beta-m)}{s}+O\left(\frac{1}{s^{2}}\right) .
\end{aligned}
$$

Hence, in order to make the series converge absolutely, it is sufficient that $\operatorname{Re}(\alpha-\beta-m)<-1$. From the condition

$$
\operatorname{Re}(\alpha-\beta-m)=\operatorname{Re}\left\{\|A\|+\left|a_{k k}\right|-\left(\rho_{j}-a_{k k}+1\right)-m\right\}<-1,
$$

we obtain $m>\|A\|-\operatorname{Re} \rho_{j}+\left|a_{k k}\right|+\operatorname{Re} a_{k k}$.

The right hand side of the above inequality is non-negative, since $\|A\| \geqq\left|\rho_{j}\right| \geqq \operatorname{Re} \rho_{j}$.

Now we choose the smallest integer $m_{0}>0$ such that

$$
m_{0}>\max _{j, h}\left\{\|A\|-\operatorname{Re} \rho_{j}+\left|a_{k k}\right|+\operatorname{Re} a_{k k}\right\} .
$$

Hence we obtain the following Theorem 1.

Theorem 1. If $\left|\lambda_{k}\right|<\left|\lambda_{j}-\lambda_{k}\right|(j \neq k)$, the series

$$
F_{j}^{k}(m)=\sum_{s=0}^{\infty} H^{k}(s) g_{j}^{k}(m+s) \quad(j=1,2, \cdots, n ; k=1,2, \cdots, n)
$$

are absolutely and uniformly convergent for all integers $m$ and satisfy the following difference equations

$$
\begin{gathered}
\left(\rho_{j}+m-A\right) F_{j}^{k}(m)=B F_{j}^{k}(m-1) . \\
\text { If }\left|\lambda_{k}\right| \leqq\left|\lambda-\lambda_{k}\right|=\min _{j \neq k}\left|\lambda_{j}-\lambda_{k}\right|, \text { only for } m \geqq m_{0}>0,
\end{gathered}
$$

the series $F_{j}^{k}(m)=\sum_{s=0}^{\infty} H^{k}(s) g_{j}^{k}(m+s)$ are absolutely convergent and satisfy the above difference equations (8).

2. In this section, using the above result, we solve the two point connection problem for a system of ordinary differential equations

$$
t \frac{d X}{d t}=(A+t B) X
$$

under the following assumptions.

( I ) For the sake of simplicity, we assume that the matrix $A$ has no congurent eigenvalues, i.e.,

$$
\rho_{j}-\rho_{k} \neq \text { integers } \quad(j=1,2, \cdots, n ; k=1,2, \cdots, n)
$$


Connection problems and the determination of Stokes' multipliers 343

(II ) $\rho_{j}-a_{k k} \neq$ non-positive integers $(j=1,2, \cdots, n ; k=1,2, \cdots, n)$

where $a_{k k}$ are the diagonal elements of the matrix $A$.

(III) The matrix $B$ is a diagonal matrix;

$$
B=\operatorname{diag}\left(\lambda_{1}, \lambda_{2}, \cdots, \lambda_{n}\right) \text {. }
$$

The eigenvalues $\lambda_{k}(k=1,2, \cdots, n)$ satisfy the following condition;

$$
0<\left|\lambda_{k}\right| \leqq\left|\lambda-\lambda_{k}\right|=\min _{j \neq k}\left|\lambda_{j}-\lambda_{k}\right| \text {. }
$$

This condition is weaker one than the pentagonal condition, because for some $k$ and $j$, the equality may hold.

The differential equations (9) have one set of fundamental solutions which are expressed in a neighbourhood of the origin by the convergent power series as follows

$$
X_{j}(t)=t_{j}^{\rho} \sum_{m=0}^{\infty} G_{j}(m) t^{m} \quad(j=1,2, \cdots, n) .
$$

Here, the coefficients $G_{j}(m)$ satisfy the following difference equations

$$
\left\{\begin{array}{l}
\left(\rho_{j}+m-A\right) G_{j}(m)=B G_{j}(m-1) \\
\left(\rho_{j}-A\right) G_{j}(0)=0 .
\end{array}\right.
$$

Theorem 2. We can determine the Stokes' multipliers $T_{j}^{k}$ $(j=1,2, \cdots, n ; k=1,2, \cdots, n)$ by solving the linear equations;

$$
G_{j}\left(m_{0}\right)=\sum_{k=1}^{n} T_{j}^{k} F_{j}^{k}\left(m_{0}\right) \quad(j=1,2, \cdots, n)
$$

and after that, we can also determine $F_{j}^{k}(m)$ for $0 \leqq m \leqq m_{0}-1$ by solving the following linear equations one after another.

$$
G_{j}(m)=\sum_{k=1}^{n} T_{j}^{k} F_{j}^{k}(m) \quad(j=1,2, \cdots, n) \quad\left(0 \leqq m \leqq m_{0}-1\right) .
$$

Then the coefficients $G_{j}(m)$ which satisfy the difference equations (11) are expressed by

$$
G_{j}(m)=\sum_{k=1}^{n} T_{j}^{k} F_{j}^{k}(m) \quad(j=1,2, \cdots, n)(m \geqq 0) .
$$

Proof. It is easy to prove that the set of $\left\{F_{j}^{k}(m): k=1,2\right.$, $\cdots, n\}(j=1,2, \cdots, n)$ constitutes an independent set of solutions of the difference equations (11) for $m \geqq m_{0}$ and that because of the assump- 
tions (I) and (II), the matrix $\left\{G_{1}(m), G_{2}(m), \cdots, G_{n}(m)\right\}$ is nonsingular.

Hence, the matrix of the Stokes' multipliers $\left\{T_{j}^{k}: j=1,2, \cdots, n\right.$; $k=1,2, \cdots, n)\}$ is non-singular. So the theorem holds evidently.

Now, before we give the asymptotic forms of the solutions $X_{j}(t)$ in the sectorial neighbourhood of the infinity, we will give some lemmas needed later.

Lemma 1. (E. M. Wright) If $\varphi(w)$ is holomorphic and bounded in the right half-plane

$$
\operatorname{Re} w \geqq h^{\prime}>0
$$

and

$$
h^{\prime}>\frac{3}{2}-\operatorname{Re} \beta,
$$

then we have

$$
\sum_{m=\left[h^{\prime}\right]+1}^{\infty} \frac{\varphi(m)}{\Gamma(m+\beta)} z^{m}=O\left(e^{z} z^{1-\beta}\right)+O\left(z^{h^{\prime}}\right)
$$

as $z$ tends to infinity in the sector

$$
|\arg z| \leqq \frac{3}{2} \pi \text {. }
$$

Here [ ] denotes Gauss' symbol.

Lemma 2. We define $h_{0}$ by

(13) $h_{0}=\max \left\{0, \frac{3}{2}+\operatorname{Re}\left(a_{k k}-\rho_{j}\right): j=1,2, \cdots, n ; k=1,2, \cdots, n\right\}$

and $q_{j, \sigma}^{k}\left(w, w_{0}: s\right)$ as follows.

$$
q_{j, \sigma}^{k}\left(w, w_{0}: s\right)=\frac{g_{j}^{k}\left(w_{0}+\sigma\right)}{g_{j}^{k}(w+\sigma)}\left\{\frac{g_{j}^{k}(w+s)}{g_{j}^{k}\left(w_{0}+s\right)}-\frac{g_{j}^{k}(w+s-1)}{g_{j}^{k}\left(w_{0}+s-1\right)}\right\}
$$

where $w_{0} \geqq m_{0}$ is an integer such that

$$
w_{0}=\sigma-\left[\frac{3}{4}+\max _{j, k}\left|\rho_{j}-a_{k k}\right|\right] .
$$

Then the series

$$
Q_{j, \sigma}^{k}\left(w, w_{0}\right)=\sum_{s=\sigma+1}^{\infty} q_{j, \sigma}^{k}\left(w, w_{0}: s\right)
$$


Connection problems and the determination of Stokes' multipliers 345 are absolutely convergent and uniformly bounded in the right halfplane

$$
\operatorname{Re} w \geqq \sigma+h_{0} .
$$

Proof. For $p \geqq w_{0}$, we have the following estimates

$$
\begin{aligned}
& \left|w+p+\rho_{j}-a_{k k}\right| \geqq \operatorname{Re}\left(w+p+\rho_{j}-a_{k k}\right) \\
& \quad \geqq \sigma+p+h_{0}+\operatorname{Re}\left(\rho_{j}-a_{k k}\right) \geqq \sigma+p+\frac{3}{2} \\
& \left|w_{0}+p+\rho_{j}-a_{k k}\right| \leqq\left|w_{0}+p\right|+\left|\rho_{j}-a_{k k}\right| \\
& \quad \leqq \sigma+p-\left[\frac{3}{4}+\max _{j, k}\left|\rho_{j}-a_{k k}\right|\right]+\left|\rho_{j}-a_{k k}\right| \leqq \sigma+p+\frac{1}{4}
\end{aligned}
$$

Then, for $s \geqq \sigma+1$, we can estimate the absolute values of $q_{j, \sigma}^{k}\left(w, w_{0}: s\right)$ as follows.

$$
\begin{aligned}
& q_{j, \sigma}^{k}\left(w, w_{0}: s\right) \\
= & \frac{\Gamma\left(w+\sigma+\rho_{j}-a_{k k}+1\right) \Gamma\left(w_{0}+s+\rho_{j}-a_{k k}\right)}{\Gamma\left(w_{0}+\sigma+\rho_{j}-a_{k k}+1\right) \Gamma\left(w+s+\rho_{j}-a_{k k}\right)}\left\{-1+\frac{w_{0}+s+\rho_{j}-a_{k k}}{w+s+\rho_{j}-a_{k k}}\right\} \\
& \left|q_{j, \sigma}^{k}\left(w, w_{0}: s\right)\right| \\
\leqq & \left|\frac{\left(w_{0}+s-1+\rho_{j}-a_{k k}\right) \cdots\left(w_{0}+\sigma+1+\rho_{j}-a_{k k}\right)}{\left(w+s-1+\rho_{j}-a_{k k}\right) \cdots\left(w+\sigma+1+\rho_{j}-a_{k k}\right)}\right|\left\{1+\frac{\left|w_{0}+s+\rho_{j}-a_{k k}\right|}{\left|w+s+\rho_{j}-a_{k k}\right|}\right\} \\
\leqq & \frac{\left(s+\sigma-\frac{3}{4}\right)\left(s+\sigma-\frac{7}{4}\right) \cdots\left(2 \sigma+\frac{5}{4}\right)}{\left(s+\sigma+\frac{1}{2}\right)\left(s+\sigma-\frac{1}{2}\right) \cdots\left(2 \sigma+\frac{5}{2}\right)}\left\{1+\frac{s+\sigma+\frac{1}{4}}{s+\sigma+\frac{3}{2}}\right\} \\
= & \frac{\Gamma\left(s+\sigma+\frac{1}{4}\right) \Gamma\left(2 \sigma+\frac{5}{2}\right)}{\Gamma\left(s+\sigma+\frac{3}{2}\right) \Gamma\left(2 \sigma+\frac{5}{4}\right)}\left\{2-\frac{1}{4}\right. \\
& \left.s+\sigma+\frac{3}{2}\right\} \\
\leqq & 2 \frac{\Gamma\left(s+\sigma+\frac{1}{4}\right) \Gamma\left(2 \sigma+\frac{5}{2}\right)}{\Gamma\left(s+\sigma+\frac{3}{2}\right) \Gamma\left(2 \sigma+\frac{5}{4}\right)}
\end{aligned}
$$

Hence, we have

$$
\left|Q_{j, \sigma}^{k}\left(w, w_{0}\right)\right| \leqq \sum_{s=\sigma+1}^{\infty}\left|q_{j, \sigma}^{k}\left(w, w_{0}: s\right)\right| \leqq 2 \sum_{s=\sigma+1}^{\infty} \frac{\Gamma\left(s+\sigma+\frac{1}{4}\right) \Gamma\left(2 \sigma+\frac{5}{2}\right)}{\Gamma\left(s+\sigma+\frac{3}{2}\right) \Gamma\left(2 \sigma+\frac{5}{4}\right)}
$$


Since the series of the right hand side is convergent, the series. $Q_{j, \sigma}^{k}\left(w, w_{0}\right)$ are absolutely convergent and uniformly bounded in the right half-plane (14).

Lemma 3. For a positive integer $\sigma$, we can define the functions $P_{j, \sigma}^{k}(w)(j=1,2, \cdots, n)$ of a complex variable $w$ by

$$
P_{j, \sigma}^{k}(w)=\frac{1}{g_{j}^{k}(w+\sigma)} \sum_{s=\sigma+1}^{\infty} H^{k}(s) g_{j}^{k}(w+s)
$$

and $P_{j, \sigma}^{k}(w)$ are holomorphic and bounded in the right half-plane $\operatorname{Re} w \geqq \sigma+h_{0}$.

Proof。 When $w_{0}$ is a positive integer and $w_{0} \geqq m_{0}$, we proved in the theorem 1 that the series $\sum_{s=0}^{\infty} H^{k}(s) g_{j}^{k}\left(w_{0}+s\right)$ are convergent and obtained the following estimation

$$
\begin{aligned}
& \left\|\sum_{s=\sigma+1}^{\infty} H^{k}(s) g_{j}^{k}\left(w_{0}+s\right)\right\| \leqq \sum_{s=\sigma+1}^{\infty}\left\|H^{k}(s) g_{j}^{k}\left(w_{0}+s\right)\right\| \\
\leqq & \frac{\left\|H^{k}(\sigma+1)\right\| \mid \Gamma(\beta-1)^{\prime}}{\Gamma(\alpha+\sigma+1)}\left|\lambda_{k}\right|^{w_{0}+\sigma+1} \sum_{s=0}^{\infty}\left\{\frac{\left|\lambda_{k}\right|}{\left|\lambda-\lambda_{k}\right|}\right\}^{s} \frac{\Gamma(s+\sigma+1+\alpha)}{\left|\Gamma\left(s+w_{0}+\sigma+1+\beta\right)\right|}=M .
\end{aligned}
$$

Then, for an arbitrary positive integer $p \geqq \sigma+1$, we obtain

$$
\left\|\sum_{s=p}^{\infty} H^{k}(s) g_{j}^{k}\left(w_{0}+s\right)\right\| \leqq M .
$$

Now, applying formally Abel's transformation to $P_{j, \sigma}^{k}(w)$, we have

$$
\begin{aligned}
P_{j, \sigma}^{k}(w) & =\frac{1}{g_{j}^{k}\left(w_{0}+\sigma\right)} \sum_{s=\sigma+1}^{\infty} H^{k}(s) g_{j}^{k}\left(w_{0}+s\right) \frac{g_{j}^{k}\left(w_{0}+\sigma\right) g_{j}^{k}(w+s)}{g_{j}^{k}(w+\sigma) g_{j}^{k}\left(w_{0}+s\right)} \\
& =\frac{1}{g_{j}^{k}\left(w_{0}+\sigma\right)} \sum_{s=\sigma+1}^{\infty} H^{k}(s) g_{j}^{k}\left(w_{0}+s\right) \sum_{p=\sigma+1}^{s} q_{j, \sigma}^{k}\left(w, w_{0}: p\right) \\
& +\frac{1}{g_{j}^{k}\left(w_{0}+\sigma\right)} \sum_{s=\sigma+1}^{\infty} H^{k}(s) g_{j}^{k}\left(w_{0}+s\right) \\
& =\frac{1}{g_{j}^{k}\left(w_{0}+\sigma\right)} \sum_{p=\sigma+1}^{\infty}\left[q_{j, \sigma}^{k}\left(w, w_{0}: p\right)\left(\sum_{s=p}^{\infty} H^{k}(s) g_{j}^{k}\left(w_{0}+s\right)\right)\right] \\
& +\frac{1}{g_{j}^{k}\left(w_{0}+\sigma\right)} \sum_{s=\sigma+1}^{\infty} H^{k}(s) g_{j}^{k}\left(w_{0}+s\right) .
\end{aligned}
$$

Here, we have 


$$
\begin{aligned}
& \sum_{p=\sigma+1}^{\infty}\left\|\left[q_{j, \sigma}^{k}\left(w, w_{0}: p\right)\left(\sum_{s=p}^{\infty} H^{k}(s) g_{J}^{k}\left(w_{0}+s\right)\right)\right]\right\| \\
& \quad \leqq \sum_{p=\sigma+1}^{\infty}\left(\left|q_{j, \sigma}^{k}\left(w, w_{0}: p\right)\right|\left\|\sum_{s=p}^{\infty} H^{k}(s) g_{j}^{k}\left(w_{0}+s\right)\right\|\right) \\
& \quad \leqq M \sum_{p=\sigma+1}^{\infty}\left|q_{j, \sigma}^{k}\left(w, w_{0}: p\right)\right| \leqq 2 M \sum_{s=\sigma+1}^{\infty} \frac{\Gamma\left(s+\sigma+\frac{1}{4}\right) \Gamma\left(2 \sigma+\frac{3}{2}\right)}{\Gamma\left(s+\sigma+\frac{3}{2}\right) \Gamma\left(2 \sigma+\frac{5}{4}\right)} .
\end{aligned}
$$

Therefore, the validity of Abel's transformation was guaranteed and we proved the absolute convergence of $P_{j, \sigma}^{k}(w)$ and also showed the uniform boundedness of $P_{j, \sigma}^{k}(w)$ in the right half-plane $\operatorname{Re} w \geqq \sigma+h_{0}$.

Lemma 4. We take a sufficiently large positive integer $m_{1}$ such that $m_{1} \geqq 2 \sigma$ and put $h=\left[h_{0}\right]+1$. Then we have

$$
\begin{aligned}
& \sum_{m=0}^{\infty} g_{j}^{k}\left(m+m_{1}+\sigma\right) P_{j, \sigma}^{k}\left(m+m_{1}\right) t^{m} \\
= & O\left(e^{\lambda_{k} t} t^{a_{k}-\rho_{j}-m_{1}-\sigma}\right)+O\left(t^{h_{0}-\sigma}\right)-\sum_{l=1}^{\sigma-k} g_{j}^{k}\left(m_{1}+\sigma-l\right) P_{j, \sigma}^{k}\left(m_{1}-l\right) t^{-l}
\end{aligned}
$$

in the sector

$$
\left|\arg \lambda_{k} t\right| \leqq \frac{3}{2} \pi
$$

Proof.

$$
\begin{aligned}
& \sum_{m=0}^{\infty} g_{j}^{k}\left(m+m_{1}+\sigma\right) P_{j, \sigma}^{k}\left(m+m_{1}\right) t^{m} \\
= & \sum_{m=l}^{\infty} g_{j}^{k}\left(m+m_{1}\right) P_{j, \sigma}^{k}\left(m+m_{1}-\sigma\right) t^{m-\sigma}-\sum_{l=1}^{\sigma-h} g_{j}^{k}\left(m_{1}+\sigma-l\right) P_{j, \sigma}^{k}\left(m_{1}-l\right) t^{-l} \\
= & \lambda_{k}^{m_{1}} t^{-\sigma} \Gamma\left(\rho_{j}-a_{k k}\right) \sum_{m=l}^{\infty} \frac{P_{j, \sigma}^{k}\left(m+m_{1}-\sigma\right)}{\Gamma\left(m+m_{1}+\rho_{j}-a_{k k}+1\right)}\left(\lambda_{k} t\right)^{m} \\
& \quad-\sum_{l=1}^{\sigma-h} g_{j}^{k}\left(m_{1}+\sigma-l\right) P_{j, \sigma}^{k}\left(m_{1}-l\right) t^{-l} .
\end{aligned}
$$

If $\operatorname{Re} w \geqq h_{0}$, we have $\operatorname{Re}\left(w+m_{1}-\sigma\right) \geqq \sigma+h_{0}$.

Hence, from the lemma 3 , it results that each component of $P_{j, \sigma}^{k}\left(w+m_{1}-\sigma\right)$ is holomorphic and uniformly bounded in $\operatorname{Re} w \geqq h_{0}$.

Since we also have $\frac{3}{2}-\operatorname{Re}\left(m_{1}+\rho_{j}-a_{k k}+1\right)<h_{0}-m_{1}-1<h_{0}$, we can apply E. M. Wright's lemma to the first term of the right hand side of the above equality and we obtain 


$$
\begin{gathered}
\lambda_{k}^{m_{1}} t^{-\sigma} \Gamma\left(\rho_{j}-a_{k k}\right) \sum_{m=h}^{\infty} \frac{P_{j, \sigma}^{k}\left(m+m_{1}-\sigma\right)}{\Gamma\left(m+m_{1}+\rho_{j}-a_{k k}+1\right)}\left(\lambda_{k} t\right)^{m} \\
=t^{-\sigma}\left\{O\left(e^{\lambda_{k} t} t^{a_{k k}-\rho_{j}-m_{1}}\right)+O\left(t^{h_{0}}\right)\right\}
\end{gathered}
$$

as $t$ tends to infinity in the sector $\left|\arg \lambda_{k} t\right| \leqq \frac{3}{2} \pi$.

Here we define the domain $\mathscr{D}_{B}$ as follows

$$
\begin{aligned}
\mathscr{D}_{k} & =\left\{t:|t| \geqq t_{0}>0, \quad\left|\arg \lambda_{k} t\right| \leqq \frac{3}{2} \pi-\eta\right\} \\
& -\left\{t: \operatorname{Re} t>-t_{0}, \quad\left|\arg \lambda_{k} t\right|>\pi\right\},
\end{aligned}
$$

where $\eta$ is an arbitrary small positive number and $t_{0}$ is a fixed positive number. (see the lemma 2.1 in the paper [2j).

Lemma 5. For $t$ in the domain $\mathscr{D}_{k}$, we have

$$
\begin{aligned}
& \sum_{m=0}^{\infty} g_{j}^{k}\left(m+m_{1}+s\right) t^{m} \\
& \quad=\lambda_{k}^{a_{k}-\rho_{j}} e^{\lambda_{k} t} t^{a_{k k}-\rho_{j}-s-m_{1}}-\sum_{l=-m_{1}+1}^{\sigma-h} g_{l}^{k}(s-l) t^{-m_{1}-l}+O\left(t^{-m_{1}-\sigma^{+} h}\right)
\end{aligned}
$$

\section{Proof.}

$$
\begin{aligned}
& \sum_{m=0}^{\infty} g_{j}^{k}\left(m+m_{1}+s\right) t^{m} \\
& \quad=t^{-m_{1}}\left[\sum_{m=0}^{\infty} g_{j}^{k}(m+s) t^{m}-\sum_{m=0}^{m_{1}-1} g_{j}^{k}(m+s) t^{m}\right] \\
& \quad=t^{-m_{1}}\left\{\lambda_{k}^{a_{k k}-\rho_{j}} e^{\lambda_{k} t} t^{a_{k k}-\rho_{j}-s}-\sum_{l=1}^{\sigma-h} g_{j}^{k}(s-l) t^{-l}+O\left(t^{h-\sigma}\right)-\sum_{m=0}^{m_{1}-1} g_{j}^{k}(m+s) t^{m}\right\} \\
& \quad=\lambda_{k}^{a_{k k}-\rho_{j}} e^{\lambda_{k} t} t^{a_{k k}-\rho_{j}-s-m_{1}}-\sum_{l=-m_{1}+1}^{\sigma-h} g_{j}^{k}(s-l) t^{-m_{1}-l}+O\left(t^{-m_{1}-\sigma+h}\right) .
\end{aligned}
$$

In the above calculation, we used the asymptotic forms of the series $\sum_{m=0}^{\infty} g_{j}^{k}(m+s) t^{m}$ which are the solutions of the fundamental equations. The method by which we derived the asymptotic forms of these series is described in detail in the papers [1] (Proposition 2.5), [2] (Lemma 2.2)

Lemma 6. For $t$ in the domain $\mathscr{D}_{k}$, we have

$$
\begin{aligned}
& \sum_{m=0}^{\infty} F_{j}^{k}\left(m_{1}+m\right) t^{m} \\
& \quad=\lambda_{k}^{a_{k k}-\rho_{j}} e^{\lambda_{k} t} t^{a_{k k}-\rho_{j}-m_{1}}\left\{\sum_{s=0}^{\sigma} H^{k}(s) t^{-s}+O\left(t^{-\sigma}\right)\right\}
\end{aligned}
$$




$$
-\sum_{l=1}^{\sigma-h} F_{j}^{k}\left(m_{1}-l\right) t^{-l}-\sum_{l=\sigma-h+1}^{\sigma-h+m_{1}}\left(\sum_{s=0}^{\sigma} H^{k}(s) g_{j}^{k}\left(s+m_{1}-l\right)\right) t^{-l}+O\left(t^{h-\sigma}\right) .
$$

Proof. We divide the above power series into two parts and apply the lemma 3 , and the lemma 4 .

$$
\begin{aligned}
& F_{j}^{k}\left(m_{1}+m\right) t^{m} \\
& =\sum_{m=0}^{\infty}\left[\sum_{s=0}^{\sigma} H^{k}(s) g_{j}^{k}\left(m+m_{1}+s\right)+g_{j}^{k}\left(m+m_{1}+\sigma\right) P_{j, \sigma}^{k}\left(m+m_{1}\right)\right] t^{m} \\
& =\sum_{s=0}^{\sigma} H^{k}(s)\left(\sum_{m=0}^{\infty} g_{j}^{k}\left(m+m_{1}+s\right) t^{m}\right)+\sum_{m=0}^{\infty} g_{j}^{k}\left(m+m_{1}+\sigma\right) P_{j \sigma}^{k}\left(m+m_{1}\right) t^{m} \\
& =\sum_{s=0}^{\sigma} H^{k}(s)\left\{\lambda_{k}^{a_{k k}-\rho_{j}} e^{\lambda_{k} t} t^{a_{k k}-\rho_{j}-m_{1}-s}-\sum_{l=-m_{1}+1}^{\sigma-h} g_{j}^{k}(s-l) t^{-m_{1}-l}\right. \\
& \left.\quad+O\left(t^{-m_{1}-\sigma+h}\right)\right\}+O\left(e^{\lambda_{k} t} t^{a_{k k}-\rho_{j}-m_{1}-\sigma}\right) \\
& \quad-\sum_{l=1}^{\sigma-h} g_{j}^{k}\left(m_{1}+\sigma-l\right) P_{j, \sigma}^{k}\left(m_{1}-l\right) t^{-l}+O\left(t^{h_{0}-\sigma}\right) \\
& =\lambda_{k}^{a_{k}-\rho_{j}} e^{\lambda_{k} t} t^{a_{k k}-\rho_{j}-m_{1}}\left\{\sum_{s=0}^{\sigma} H^{k}(s) t^{-s}+O\left(t^{-\sigma}\right)\right\} \\
& \quad-\left\{\sum_{l=1}^{\sigma-h} g_{j}^{k}\left(m_{1}+\sigma-l\right) P_{j, \sigma}^{k}\left(m_{1}-l\right) t^{-l}\right. \\
& \left.\quad+\sum_{l=-m_{1}+1}^{\sigma-h}\left(\sum_{s=0}^{\sigma} g_{j}^{k}(s-l) H^{k}(s)\right) t^{-m_{1}-l}\right\}+O\left(t^{h-\sigma}\right) .
\end{aligned}
$$

Here we have

$$
\begin{aligned}
& \sum_{l=1}^{\sigma-h} g_{j}^{k}\left(m_{1}+\sigma-l\right) P_{j, \sigma}^{k}\left(m_{1}-l\right) t^{-l}+\sum_{l=-m_{1}+1}^{\sigma-h}\left(\sum_{s=0}^{\sigma} g_{j}^{k}(s-l) H^{k}(s)\right) t^{-m_{1}-l} \\
& =\sum_{l=1}^{\sigma-h} g_{j}^{k}\left(m_{1}+\sigma-l\right) P_{j, \sigma}^{k}\left(m_{1}-l\right) t^{-l}+\sum_{l=1}^{m_{1}+\sigma-h}\left(\sum_{s=0}^{\sigma} g_{j}^{k}\left(s+m_{1}-l\right) H^{k}(s)\right) t^{-l} \\
& =\sum_{l=1}^{\sigma-h} F_{j}^{k}\left(m_{1}-l\right) t^{-l}+\sum_{l=\sigma-h+1}^{m_{1}+\sigma-h}\left(\sum_{s=0}^{\sigma} H^{k}(s) g_{j}^{k}\left(s+m_{1}-l\right)\right) t^{-l} .
\end{aligned}
$$

Hence we obtain the lemma 5 .

Now we will show the asymptotic forms of the convergent solutions of the differential equations (9).

Theorem 3. For $t$ in the domain $\mathscr{D}=\bigcap_{k=1}^{n} \mathscr{D}_{k}$, we have for $j=1$, $2, \cdots, n$,

$$
\begin{aligned}
& X_{j}(t)=\sum_{k=1}^{n} T_{j}^{k} \lambda_{k}^{a_{k k}-\rho_{j}} e^{\lambda_{k} t} t^{a_{k k}}\left\{\sum_{s=0}^{\sigma} H^{k}(s) t^{-s}+O\left(t^{-\sigma}\right)\right\} \\
& \quad+\sum_{k=1}^{n} T_{j}^{k}\left(\sum_{m=0}^{m_{1}-\sigma+h-1} F_{j}^{k}(m) t^{m+\rho_{j}}-\sum_{m=-\sigma+h}^{m_{1}-\sigma+h-1}\left(\sum_{s=0}^{\sigma} H^{k}(s) g_{j}^{k}(s+m)\right) t^{m+\rho_{j}}\right) \\
& \quad+O\left(t^{m_{1}+\rho_{j}+h-\sigma}\right) .
\end{aligned}
$$


In particular, if there is at least one index $k$, for which $\operatorname{Re}\left(\lambda_{k} t\right)$ is non-negative in the domain $\mathscr{D}$, then we obtain

$$
\begin{aligned}
X_{j}(t) \cong & \sum_{k=1}^{n} T_{j}^{k} \lambda_{k}^{a_{k}-\rho_{j}} e^{\lambda_{k} t} t^{a_{k k}}\left\{\sum_{s=0}^{\sigma} H^{k}(s) t^{-s}+O\left(t^{-\sigma}\right)\right\} \\
& (j=1,2, \cdots, n) .
\end{aligned}
$$

Proof. Using the theorem 2, we decompose the convergent solutions as follow's.

$$
\begin{aligned}
X_{j}(t) & =t^{\rho_{j}} \sum_{m=0}^{\infty} G_{j}(m) t^{m}=\sum_{k=1}^{n} T_{j}^{k}\left(\sum_{m=0}^{\infty} F_{j}^{k}(m) t^{m^{+\rho_{j}}}\right) \\
& =\sum_{k=1}^{n} T_{j}^{b}\left(\sum_{m=0}^{m_{1}-1} F_{j}^{k}(m) t^{m+\rho_{j}}+\sum_{m=0}^{\infty} F_{j}^{k}\left(m+m_{1}\right) t^{m+m_{1}+\rho_{j}}\right) .
\end{aligned}
$$

Here we apply the lemma 5 to the second part of the right hand side, and we have

$$
\begin{aligned}
X_{j}(t) & =\sum_{k=1}^{n} T_{j}^{k}\left[\lambda_{k}^{a_{k k}-\rho_{j}} e^{\lambda_{k} t} t^{a_{k k}}\left\{\sum_{s=0}^{\sigma} H^{k}(s) t^{-s}+O\left(t^{-\sigma}\right)\right\}\right. \\
& +\sum_{m=0}^{m_{1}-1} F_{j}^{k}(m) t^{m+\rho_{j}}-\sum_{l=1}^{\sigma-h} F_{j}^{k}\left(m_{1}-l\right) t^{m_{1}-l+\rho_{j}} \\
& \left.-\sum_{l=\sigma-h+1}^{\sigma-h+m_{1}}\left(\sum_{s=0}^{\sigma} H^{k}(s) g_{j}^{k}\left(s+m_{1}-l\right)\right) t^{m_{1}-l+\rho_{j}}+O\left(t^{m_{1}+\rho_{j}+h-\sigma}\right)\right] \\
= & \sum_{k=1}^{n} T_{j}^{k} \lambda_{k}^{a_{k}-\rho_{j}} e^{\lambda_{k} t} t^{a_{k k}}\left\{\sum_{s=0}^{\sigma} H^{k}(s) t^{-s}+O\left(t^{-\sigma}\right)\right\} \\
& +\sum_{k=1}^{n} T_{j}^{k}\left\{\sum_{m=0}^{m_{1}-\sigma+h-1} F_{j}^{k}(m) t^{m+\rho_{j}}-\sum_{m=-\sigma+h}^{m_{1}-\sigma+h+1}\left(\sum_{s=0}^{\sigma} H^{k}(s) g_{j}^{k}(m+s)\right) t^{m+\rho_{j}}\right\} \\
& +O\left(t^{m_{1}+\rho_{j}+h-\sigma}\right) .
\end{aligned}
$$

When the matrix $A$ has any congruent eigenvalues, the same results will be obtained.

The auther would like to express his sincere thanks to Professor M. Hukuhara for his kind guidance and warm encouragement.

\section{REFERENCES}

[1] Okubo, K., A connection problem involving a logarithmic function, Publ. RIMS, Kyoto Univ. Ser. A, 1 (1965), 99-128.

[2] Kohno, M., A two points connection problem involving logarithmic polynomials, Ibid. 2 (1966), 269-305.

[3] Iwano, M. and K. Okubo, On a set of convergent solutions for a system of second order linear difference equations, Ibid. 1 (1966), 205-210. 